\title{
Impact of perioperative treatment on survival of resectable gastric cancer patients after D2 lymphadenectomy: a single European centre propensity score matching analysis
}

\author{
Tomaz Jagric ${ }^{1}$, Bojan Ilijevec¹, Vaneja Velenik², Janja Ocvirk², Stojan Potrc \\ ${ }^{1}$ Department for Abdominal and General Surgery, University Clinical Centre Maribor, Maribor, Slovenia \\ ${ }^{2}$ Institute of Oncology Ljubljana, Ljubljana, Slovenia
}

Radiol Oncol 2019; 53(2): 245-255.

Received 12 December 2018

Accepted 24 February 2019

Correspondence to: Tomaž Jagrič, M.D., Ph.D., Department for Abdominal and General Surgery, University Clinical Centre Maribor, Ljubljanska ulica 5, 2000 Maribor, Slovenia. Phone: +386-23211301; Fax: +386-23211262; E-mail: tomaz.jagric@gmail.com

Disclosure: No potential conflicts of interest were disclosed.

\begin{abstract}
Background. To determine the effects of perioperative treatment of gastric cancer patients, we conducted an analysis with propensity score matched patient groups to determine the role of perioperative chemotherapy in patients after D2 lymphadenectomy.

Patients and methods. From our database of 1563 patients, 482 patients were selected with propensity score matching and divided into two balanced groups: 241 patients in the surgery only group and 241 patients in the perioperative group. The long-term results of treatment were compared between the two groups.

Results. Most of the included patients received radio-chemotherapy with capecitabine $(n=111 ; 46 \%)$ and perioperative chemotherapy with epirubicin, oxalliplatin and capecitabine $(n=91 ; 37.7 \%) .92 .9 \%$ of the patients received a D2 lymph node dissection. Perioperative morbidity was similar between surgery only (18.3\%) and perioperative treatment groups $(20.7 \%)(p=0.537)$. The perioperative mortality was not influenced by perioperative treatment. A pathological response was observed in $12.5 \%$ of patients. The overall 5 -year and median survivals were significantly higher in the perioperative treatment group (50.5\%; 51.7 moths) compared to surgery only group (41.8\%; 34.9 months; $p=0.038$ ). The subgroup analysis revealed that only patients with the TNM stages T3 $(p=0.028), N 2(p=0.009)$, N3b $(p=0.043)$, and UICC stages IIII $(p=0.003)$ and IIIC $(p=0.03)$ significantly benefit from perioperative treatment.

Conclusions. Perioperative treatment in radically resected gastric cancer patients after D2 lymphadenectomy was beneficial in stages Illb and Illc. The effects of perioperative treatment in lower stages could be negated by the effects of the radical surgery in lower stages and in higher stages by the biology of the disease.
\end{abstract}

Key words: perioperative treatment; gastric cancer; D2 lymphadenectomy; propensity score matching

\section{Introduction}

Multimodal treatment has long been established as the only way to prolong the poor survival of patients with advanced gastric cancer. ${ }^{1-13}$ With this therapy, long term survival has increased from $38 \%$ to $70 \%{ }^{1-13}$ Chemotherapy is now a solid part of gastric cancer treatment guidelines, but there is still much debate on which regimen should be used, the time and duration of chemotherapy.,
Before INT0116 trial, gastric cancer was supposed to be chemoresistant. The INT0116 trial was one of the first trials that established the adjuvant chemoradiotherapy. 1,2,14 The study influenced the treatment in Northern America. ${ }^{1,2}$ Although it clearly demonstrated the effectiveness of the chemoradiotherapy in gastric cancer, the major concern was that it was carried out on patients with suboptimal lymphadenectomy. ${ }^{1,2}$ It is now accepted that this protocol improves survival in suboptimal oper- 
ated patients. ${ }^{1,2}$ In the following years, the ACSTGC trial, and later the Sakato's trial conducted in Japan, proved beyond any doubt that adjuvant treatment can improve survival even in patients after adequate lymphadenectomy. ${ }^{6}$ But the results from FLAGS trial showed that the tolerance of S1 agent in Caucasian population was poor, and only a fraction of patients with esophago-gastric junction were included. ${ }^{2}$ Meanwhile, in Europe the results of the MAGIC trial proved the efficiency of perioperative chemotherapy. ${ }^{3}$ Nonetheless, a sufficient lymph node dissection was performed only in $40 \%$ of patients and only $40 \%$ of patients could successfully end all postoperative cycles. In spite of the important results, there are still many trials that try to determine the best chemotherapy timing in adequate operated patients. ${ }^{3}$

In our institution, patients have been operated according to Japanese guidelines since $1992 .{ }^{15}$ We started to use the perioperative treatment in 2003. Theoretically, the study of patients from single institution where only five dedicated surgeons perform a standardised operation provides a homogenous group on which one could easily determine the beneficial effects of (perioperative and adjuvant) chemotherapy. Therefore, we conducted an analysis with propensity score matched patients to determine the role of perioperative and adjuvant chemotherapy in patients after D2 lymphadenectomy.

\section{Patients and methods}

\section{Patients}

Since 1991, 1563 patients were operated for gastric cancer in the department for Abdominal and General Surgery at the University Clinical Centre Maribor, Slovenia. The demographic characteristics of patients, the characteristics of the surgical procedures, and the pathological characteristics of tumours were prospectively stored on a computer database. The bone marrow, renal and hepatic functions are important determinants of chemotherapeutical treatment, those factors were not routinely stored in our database. Although this might have brought a certain bias into the analysis that is inherent to retrospective studies, we assumed that unfit patients would not have been operated in the first place. Since 1991 there have been several revisions of the The Union for International Cancer Control Tumour Node Metastases classification (UICC TNM) classification. We have therefore been regularly updating the TNM classification to con- cur with the most current issue of the UICC TNM classification system. The survivals were annually updated with the data from the National Cancer Registry of Slovenia to obtain the most accurate survival data and to avoid losing any patient during follow-up. The perioperative treatment of gastric cancer patients was adopted based on the published results of the MAGIC trial from the year 2003. ${ }^{14}$ At first, patients were treated with the 5FU-LV (5-Fluorouracil-Leucovorin) protocol; but shortly after the results of the OE trial, this protocol was replaced with other chemotherapy regimens that are better tolerated by the patients. ${ }^{11}$ Now, the most used chemotherapy regimens are epirubicin, oxaliplatine and capecitabine. More than half of patients in whom perioperative treatment was instituted received one of the formal chemotherapy regimens. To determine the efficiency of perioperative and adjuvant chemotherapy in patients from a European centre after formal D2 lymph node dissection, the prospectively stored data from our patients was used. For propensity score matching, only patients with histologically verified adenocarcinoma were included. Furthermore, only patients with a R0 resection were included in the study. Patients with a metastatic disease at presentation were excluded from the study. After exclusion, 1156 patients were used for propensity score matching. Age, tumour site, complication stage according to Dindo-Claviene classification, UICC stage and the TNM nodal stage were determined to be the significant covariates for perioperative and adjuvant chemotherapy with logistic regression. Based on these covariates, a propensity score was calculated for each patient. Patients were randomised and paired with the nearest score matching protocol. In the final group, 482 patients remained. These were used for further analysis.

The study was conducted according to the ethical directives of the Helsinki declaration. All of the patients gave their informed consent before treatment. All of the patient data was stored prospectively in the hospital database, and the study was approved by local Ethnics Committee.

\section{Treatment}

The eligibility criteria for perioperative treatment were as follows: Resected gastric cancer stage IIA or higher and no distant metastases. Patients with IB gastric cancer were reviewed at tumour board for consideration of perioperative and adjuvant therapy. Further eligibility criteria were age 18 years or older, Eastern Cooperative Oncology 
Group (ECOG) 0 to 1, and adequate hepatic, renal, marrow and cardiac function. Patients with a history of recent myocardial ishaemia, uncontrolled angina, hypertension, cardiac arrhythmias, congestive heart failure, or other serious medical illness were taken under review from the tumour board. The exclusion criteria for perioperative and adjuvant treatment were as follows: Stage IA or IB (T2aN0) disease, microscopically positive resection margins, and involvement of M1 lymph node or distant metastases. Severe renal impairment (calculated creatinine clearance less than $30 \mathrm{~mL} / \mathrm{min}$ ) suspected dihydropyrimidine dehydrogenase (DPD) deficiency.

Perioperative and adjuvant treatment was administered as described elsewhere. ${ }^{16}$ In brief; patients received one of the following perioperative protocols: capecitabine, EOX (epirubicin, oxaliplatin, capecitabine), XELOX (capecitabine, oxaliplatin). Patients subjected to EOX regimen received intravenous bolus of epirubicin at a dose of $50 \mathrm{mg}$ per square meter, oxaliplatin at a dose of $130 \mathrm{mg}$ per square meter and capecitabine at a dose of 1000 mg per square meter twice a day. Treatment was repeated every 3 weeks for maximum of six cycles. The XELOX regimen consisted of eight 3-week cycles of oral capecitabine $1000 \mathrm{mg}$ per square meter twice a day on days 1 to 14 of each cycle and intravenous oxaliplatin $130 \mathrm{mg}$ per square meter on day one of each cycle. Adjuvant treatment with capecitabine was initiated within 6-8 weeks after surgery and consisted of concomitantly applied chemo- and radiotherapy. Chemotherapy started with peroral capecitabine $1250 \mathrm{mg} / \mathrm{m} 2$ twice a day (bid) on days $1-14$, with a one-week break. Concurrently with irradiation, continuous capecitabine $825 \mathrm{mg} / \mathrm{m} 2$ bid was administered, without weekend breaks. After the completion of radiotherapy with two-week break, the patients received three more cycles of capecitabine $1250 \mathrm{mg} / \mathrm{m} 2$ bid on days 1-14, with a one-week break between each cycle. Patients were irradiated on linear accelerator with $15 \mathrm{MV}$ photon beams for five days per week, at a daily dose of $1.8 \mathrm{~Gy} .{ }^{16} \mathrm{~A}$ minority of patients received either 5-fluorouracil (5FU) and cisplatin or paclitaxel and docetaxel regimen. Patients received 75 to 1000 $\mathrm{mg}$ cisplatin per square meter as intravenous infusion on day one and 29 in the 5FU-CP (5-fluorouracil and cisplatin) group, and 750 to $1000 \mathrm{mg}$ per square meter as continuous infusion over 24 hours on days one to 4 and 29 to 32 in the 5FU. Patients were irradiated on linear accelerator with $15 \mathrm{MV}$ photon beams for five days per week, at a daily dose of $1.8 \mathrm{~Gy} .{ }^{16}$ In the paclitaxel and docetaxel group, patients received 135 to $250 \mathrm{mg}$ paclitaxel per square meter as intravenous infusion every 21 days and 75 to $100 \mathrm{mg}$ docetaxel per square meter as continuous infusion every 21 days.

Gastric cancer surgery in our institution is performed by five dedicated gastric cancer surgeons. These surgeons follow the Japanese Gastric Cancer guidelines (JGCC). In well differentiated cancers located in the distal third of the stomach, for the proximal border a two to three $\mathrm{cm}$ safety margin from the palpable tumour edge is used. In these patients, a distal subtotal gastrectomy is performed, with the distal margin at least $1.5 \mathrm{~cm}$ distal to pylorus. In moderately and poor differentiated tumours, a wider resection margin of four to six $\mathrm{cm}$ is used. In these patients, a total gastrectomy is usually performed. In patients with middle third gastric cancer, a total gastrectomy is performed. In proximal third tumours and tumours of the esophagogastric junction Siewert II and Siewert III classificaton, a trans-hiatal extended total gastrectomy and distal esophagectomy or a proximal gastrectomy are performed. A pancreas preserving D2 lymphadenectomy is always performed. According to guidelines, the lymph node stations $1,3,4,5,6,7$, $8,9,11 \mathrm{p}$ and 12 are removed during a distal subtotal gastrectomy. During a total gastrectomy, the lymph node stations 1 to 12 and the left paraaortic lymph nodes are dissected. Additionally to formal lymph node stations, periesophageal lower and middle lymph nodes are dissected (lymph node stations 110 and 111) in transhiatally extended resections. The pancreatic tail is resected only if direct invasion from the tumour is present. Similarly, a splenectomy is performed only if direct invasion is present or if injury to the spleen should occur during the operation. It has been long established that splenectomy does not have an impact on longterm survival nor is splenectomy with gastrectomy considered multivisceral resection. ${ }^{17}$ These operations were therefore considered simple resections with D2 lymphadenectomy.

\section{Propensity score matching}

Patients in the surgery only and perioperative and adjuvant treatment groups were matched using the propensity score method as described by Rosenbaum and Rubin. ${ }^{18,19}$ First, the correlations between different covariates and the likelihood receiving perioperative and adjuvant treatment were analysed. The propensity score for an individual was calculated on the given covariates of preoperative serum haemoglobin levels, distal 
resection border, lymphocyte infiltration and the TNM N stage using the multivariate logistic regression model. Because radically resected gastric cancer patients in whom D2 lymphadenectomy was performed were included in the study, lymph node dissection and resection margins were not considered as a covariates in the propensity score derivation model. Using the propensity scores, 241 surgery only patients were individually matched to 241 patients who received perioperative and adjuvant treatment using the technique of the nearest available score matching. This method consists of randomly ordering the case and control subjects, then selecting the first case subject and finding the control subject with the closest propensity score. Both subjects are then manually removed from the consideration for matching and the next case subject is selected.

\section{Follow-up}

Follow-up was carried out by surgeons and oncologists. Patients underwent regular clinical assessments and laboratory testing with tumour marker determination (CEA, Ca 19-9, and Ca 72-4 from the year 2012) and abdominal ultrasound every three months for the first two years, then every six months for three years, and yearly afterwards until death. After one year, every patient had a routine upper gastro-intestinal endoscopy and chest $\mathrm{X}$-ray. In case of recurrence suspicion, additional computer tomography imaging or positron emission computer tomography was performed. Barium studies were performed in case of dysphagia.

The presence of a relapse was determined by means of imaging studies, including computer tomography, or in doubtful and inconclusive cases after negative computer tomography in patients with elevated tumour markers or high suspicion for recurrence, positron emission tomography or magnetic resonance imaging was performed. If a recurrence was detected, the patient was discussed on a tumour board to determine wheatear a palliative surgical procedure, palliative oncological treatment, or best supportive care should be commended for the patient.

\section{Outcomes}

Primary end-point of the analysis was the 5-year overall survival and the median survival. Survival was defined as the time from the operation to the death from any cause. Secondary end-points of the study were the causes of death, prediction of response, prediction of the effect of perioperative and adjuvant treatment on perioperative morbidity and mortality, and the analysis of the tumour recurrence sites. Other secondary end-points were the correlations between disease recurrence and tumour TNM stage.

\section{Statistical analysis}

Based on the results of the MAGIC trial, a 5-year survival in the perioperative and adjuvant chemotherapy group was expected to be $50 \%$ and $35 \%$ in the surgery only group. To achieve a statistical power of $80 \%$ to detect an effect at $\alpha$ level of $5 \%$, at least 161 patients were needed in each group. Continuous variables were expressed as mean \pm SD and categorical variables as percentage. Continuous variables were compared with Student's t-test for normally distributed variables; nonparametric variables were tested with MannWhitney's U-test. Normality was tested with means of Q-Q plots. The correlations between variables were tested with Pearson's bivariate correlation test, Chi square test and Student's t-test. Variables above the threshold $p$ value of 0.1 were included for multivariate analysis. The Cox regression model was used for primary analysis and included covariates that had a $p$ value of more than 0.1 in univariate analysis. Estimates of treatment effect were expressed as hazard ratios with $95 \%$ confidence interval. Kaplan-Meier curves were constructed to determine time-to-event end-points. Differences in survivals between groups were determined with the Log-rank and Breslow tests. P value of $>0.05$ was selected as the level of significance. All statistical analyses were performed on SPSS for Windows 10 v. 22 (IBM).

\section{Results}

Four hundred eighty-two patients operated between years 1991 and 2018 were included in the study. Half of the patients $(n=241)$ were treated with surgery only, while the other half of the included patients $(n=241)$ received perioperative radio-chemotherapy/chemotherapy and adjuvant chemotherapy. Patient demographic and tumour characteristics were well balanced between the two groups (Table 1).

Since 2003, when perioperative and adjuvant treatment became the standard for gastric cancer patients, different types of chemotherapy regimens were used. However, the main bulk of included 
TABLE 1. Clinicopathological characteristics of the gastric cancer patients

\begin{tabular}{|c|c|c|c|c|}
\hline \multirow{2}{*}{ Clinicopathological characteristic } & All patients & Surgery only & Perioperative and adjuvant treatment & \multirow{2}{*}{$\mathbf{P}$} \\
\hline & $(n=482)$ & $(n=241)$ & $(n=241)$ & \\
\hline Age [years $\pm S D$ ] & $62.2 \pm 11.2$ & $62.02 \pm 12.3$ & $62.35 \pm 9.9$ & NS \\
\hline Gender $[n(\%)]$ & & & & NS \\
\hline Male & $322(66.8)$ & $153(63.5)$ & $169(70.1)$ & \\
\hline Female & $160(33.2)$ & $88(36.5)$ & $72(29.9)$ & \\
\hline Type of chemotherapy [n (\%)]* & & & $\begin{array}{c}\text { Capecitabine } 111(46) \\
\text { EOX } 91(37.7) \\
\text { XELOX 16(6.6) } \\
\text { 5FUCP 19(7.9) } \\
\text { 5FULV 3(1.2) } \\
\text { Paclitaxel+CP 1(0.4) }\end{array}$ & \\
\hline \multicolumn{5}{|l|}{ Tumor site } \\
\hline Distal third & $151(31.3)$ & $75(31.1)$ & $76(31.5)$ & \multirow{5}{*}{ NS } \\
\hline Middle third & $226(46.9)$ & $113(46.9)$ & $113(46.9)$ & \\
\hline Proximal third & $85(17.6)$ & $38(15.8)$ & $47(19.5)$ & \\
\hline Whole stomach & $11(2.3)$ & $7(2.9)$ & $4(1.7)$ & \\
\hline Stump & $9(1.9)$ & $8(3.3)$ & $1(0.4)$ & \\
\hline ASA score [n (\%)] & & & & 0.044 \\
\hline । & $188(39)$ & $90(37.3)$ & $98(40.7)$ & \\
\hline$\|$ & $239(49.6)$ & $113(46.9)$ & $126(52.3)$ & \\
\hline III & $55(11.4)$ & $38(15.8)$ & $17(7.1)$ & \\
\hline T stage $[\mathrm{n}(\%)]$ & & & & NS \\
\hline TO & $6(1.2)$ & $2(0.8)$ & $4(1.7)$ & \\
\hline TI & $62(12.9)$ & $36(14.9)$ & $26(10.8)$ & \\
\hline T2 & $75(15.6)$ & 35 (14.5) & $40(16.6)$ & \\
\hline T3 & $266(55.2)$ & $138(57.3)$ & $128(53.1)$ & \\
\hline $\mathrm{T} 4$ & $73(15.1)$ & $30(12.4)$ & $43(17.8)$ & \\
\hline $\mathrm{N}$ stage [n (\%)] & & & & NS \\
\hline NO & $158(32.8)$ & $86(35.7)$ & $72(29.9)$ & \\
\hline NI & $82(17)$ & 31 (12.9) & $51(21.2)$ & \\
\hline N2 & $97(20.1)$ & $42(17.4)$ & $55(22.8)$ & \\
\hline N3a & 89 (18.5) & $53(22)$ & $36(14.9)$ & \\
\hline N3b & $56(11.6)$ & $29(12)$ & $27(11.2)$ & \\
\hline \multicolumn{5}{|l|}{ UICC stage [n (\%)] } \\
\hline 0 & $8(8)$ & $1(0.4)$ & $3(1.2)$ & \multirow{10}{*}{ NS } \\
\hline la & $54(11.2)$ & 35 (14.5) & $19(7.9)$ & \\
\hline $\mathrm{lb}$ & $35(7.3)$ & $16(6.6)$ & $19(7.9)$ & \\
\hline$\| a$ & $73(15.1)$ & $36(14.9)$ & $37(15.4)$ & \\
\hline $11 \mathrm{~b}$ & $83(17.2)$ & $30(12.4)$ & $53(22)$ & \\
\hline$\| c$ & $2(0.4)$ & $2(0.8)$ & $0(0)$ & \\
\hline Illa & $94(19.5)$ & 44 (18.3) & $50(20.7)$ & \\
\hline IIllb & $79(16.4)$ & 47 (19.5) & $32(13.3)$ & \\
\hline IIIC & $57(11.8)$ & $30(12.4)$ & $27(11.2)$ & \\
\hline IV & $1(0.2)$ & $0(0)$ & $1(0.4)$ & \\
\hline \multicolumn{5}{|l|}{ Clavien-Dindo classification [n (\%)] } \\
\hline 0 & $388(80.5)$ & $197(81.7)$ & $191(79.3)$ & NS \\
\hline । & $0(0.0)$ & $0(0)$ & $0(0)$ & \\
\hline$\|$ & $44(9.1)$ & $14(5.8)$ & $30(12.4)$ & \\
\hline Illa & $10(2.1)$ & $3(1.2)$ & $7(2.9)$ & \\
\hline IIllb & $27(5.6)$ & $16(6.6)$ & $11(4.6)$ & \\
\hline IV & $0(0)$ & $0(0)$ & $0(0)$ & \\
\hline V & $13(2.7)$ & $11(4.6)$ & $2(0.8)$ & \\
\hline Number of extracted LNs $[n \pm S D]$ & $25.6 \pm 13.1$ & $24.9 \pm 13.5$ & $26.3 \pm 12.6$ & NS \\
\hline Number of positive LNs $[\mathrm{n} \pm \mathrm{SD}]$ & $6.2 \pm 9.3$ & $6.8 \pm 10.5$ & $5.6 \pm 8$ & NS \\
\hline $\begin{array}{l}\text { Tumour diameter } \\
{[\mathrm{mm} \pm \mathrm{SD}]}\end{array}$ & $59.4 \pm 35.8$ & $61.4 \pm 38.6$ & $57.6 \pm 33.3$ & NS \\
\hline CEA $[\mu \mathrm{g} / \mathrm{I} \pm \mathrm{SD}]$ & $5 \pm 14.5$ & $4.25 \pm 10.9$ & $5.7 \pm 16.9$ & NS \\
\hline CA $19-9[\mu \mathrm{U} / \mathrm{I} \pm \mathrm{SD}]$ & $135.1 \pm 799.5$ & $66.8 \pm 287.8$ & $189.9 \pm 1037.3$ & NS \\
\hline Perioperative morbidity [\%] & $9(33.3)$ & $2(28.6)$ & $7(35)$ & NS \\
\hline Mortality [\%] & $0(0)$ & $0(0)$ & $0(0)$ & NS \\
\hline
\end{tabular}

** = significance was not determined, because only one group recived chemotherapy; ASA = American Society of Anesthesiologists; EOX = Epirubicin, Oxaliplatin, Capecitabine; LN = lymph nodes. NS = no significant difference between surgical procedures; UICC = The Union for International Cancer Control Tumour Node Metastases classification; XELOX = capecitabine, oxaliplatin; 5FUCP = 5-fluorouracil and cisplatin; paclitaxel+CP = paclitaxel and cisplatin: 5FULV = 5-fluorouracil and leucovorin 
patients received only two types of treatment: [i] radio-chemotherapy with capecitabine ( $\mathrm{n}=111$; $46 \%$; and [ii] perioperative chemotherapy with epirubicin, oxalliplatin and capecitabine ( $\mathrm{n}=91$; $37.7 \%$ ). The remaining types of chemotherapy or radio-chemotherapy regimens were applied only in less than $20 \%$ (Table 1 ). The perioperative treatment was completed in $71 \%$ of cases. Only $22.8 \%$ of perioperatively patients reported complications associated with their chemotherapy regimen. After preoperative treatment, $76.3 \%$ of patients proceeded to surgery and received adjuvant treatment. From these patients $(n=184), 93 \%$ completed the adjuvant treatment, with $7 \%$ patients who did not complete treatment because of location, size, depth of invasion, Lauren type, chemotherapy toxicity, poor general condition, tumour progression and noncompliance.

After preoperative chemotherapy/radio-chemotherapy, patients proceeded to surgery. A curative resection was achieved in all cases. The type of resection was dependant on the tumour; $46.9 \%$ ), total gastrectomy was performed in the majority of patients $(n=331 ; 64.5 \%)$. The second most prominent tumour location was the distal third of the stomach $(n=151 ; 31.3 \%)$. From 85 patients $(17.6 \%)$ with the tumour in the proximal third, a transhiatally extended total gastrectomy with resection of the distal esophagus had to be performed in 48 patients $(10 \%)$. In our hospital, all patients, excluding those with early gastric cancer, are treated with extensive D2 lymphadenectomy with the preservation of the pancreatic tail and spleen. From the included patients in this study, 92.9\% received a D2 lymph node dissection. Perioperative complications occurred in 94 patients (19.5\%). According to Claviene-Dindo classification, 9.1\% $(\mathrm{n}=44)$ were grade II, $2.1 \%(n=10)$ were grade IIIa, and 5.6\% (n $=27$ ) were grade IIIb. The cumulative perioperative mortality was $1.2 \%$.

Perioperative morbidity was similar between surgery only (18.3\%) and perioperative and adjuvant treatment group $(20.7 \%)(p=0.537)$. Although patients treated with perioperative radio-chemotherapy/chemotherapy had more grade II complications $(12.4 \%$ in the perioperative and adjuvant group vs. $5.8 \%$ in the surgery group), and patients in the surgery only group had more grade $\mathrm{IIIb}$ complications $(4.6 \%$ in the perioperative and adjuvant group vs. $6.6 \%$ in the surgery group), there was no significant difference in the distribution of the grade of complications between both groups ( $p$ $=0.537$ ) (Table 1). Surgical and general complications were also equally distributed in both groups
(Table 1). The perioperative mortality in the surgery only group was $2.2 \%$ and $0.4 \%$ in the perioperative and adjuvant treatment group.

The pathological characteristics of the tumour were balanced between groups (Table 1). Most of the tumours were poorly differentiated $(55.9 \%$ in surgery only vs. $53.2 \%$ in the perioperative and adjuvant treatment group; $\mathrm{p}=0.696)$, intestinal type $(44.9 \%$ surgery only vs. $51.6 \%$; $\mathrm{p}=0.644)$, without lymphocyte infiltration, vascular invasion, extranodal invasion and with perineural invasion (Table 1). The UICC and TNM stage distribution was similar between both groups (Table 1). Most of the patients had either IIb stage $(13.2 \%$ in surgery only vs. $22 \%$ in perioperative and adjuvant group) or IIIb stage $(18.3 \%$ in surgery only vs. $20.7 \%$ in perioperative and adjuvant treatment group; $\mathrm{p}=$ 0.954). The depth of invasion was mostly into subserosal layer in both groups (57.3\% surgery only vs. $53.1 \%$ perioperative and adjuvant treatment group; $\mathrm{p}=0.254$ ), and only a third of the patients had a node negative disease (Table 1 ). Pathological response was estimated based on the number of patients with a complete response and patients with T1 tumours. Since patients with T1 tumours were not eligible for perioperative treatment, we assumed that higher stage tumours were downsized to T1 stage. A complete pathological response was observed in $1.7 \%$ of patients in the perioperative and adjuvant treatment group and $10.8 \%$ of patients had a partial response. Counted together, a downsizing was achieved in $12.5 \%$ of patients. The mean number of extracted lymph nodes per operation was balanced between groups (surgery only: $24.9 \pm 13.5$ lymph nodes per operation $v$ s. perioperative and adjuvant therapy group: $26.3 \pm 12.6$ lymph nodes per operation; $\mathrm{p}=0.252$ ).

After a mean follow-up of 50.6 months (95\% CI: $45.4-55.8), 55 \%(n=132)$ of patients in the surgery only and $49.6 \%(n=119)$ in the perioperative and adjuvant treatment group had died. In $23(9.2 \%$ of the diseased patients) diseased patients, an autopsy was performed to determine the cause of death. Causes of death for these patients are listed in Table 1. The most common recurrence site in the autopsied patients was the peritoneal cavity (52\%), followed by haematogenous spread $(47 \%)$. The most common sites of hemathogenic recurrence were liver, lungs, adrenal glands and bone metastases listed in descending frequency (Table 2). Though the recurrence was determined only in $9.2 \%$ of diseased patients, we believe that the distribution of recurrence site in this group mirrors the actual recurrence sites in the 251 diseased patients. 


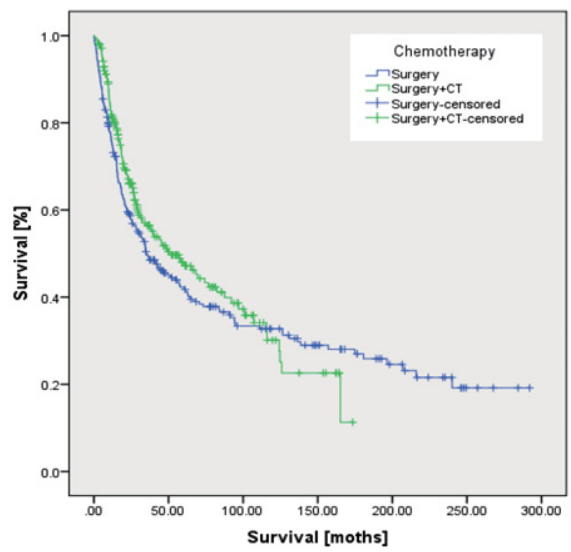

FIGURE 1. Overall survival of patients in Surgery compared to Surgery with chemotherapy group.

Surgery+CT = surgery with chemotherapy group

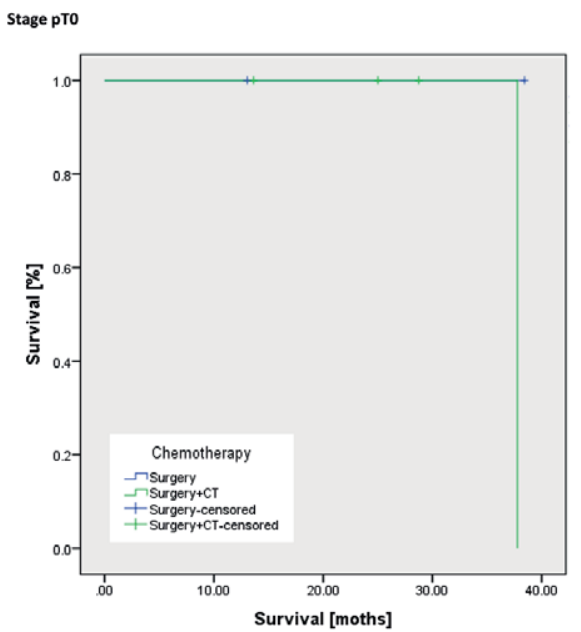

Stage p p 3

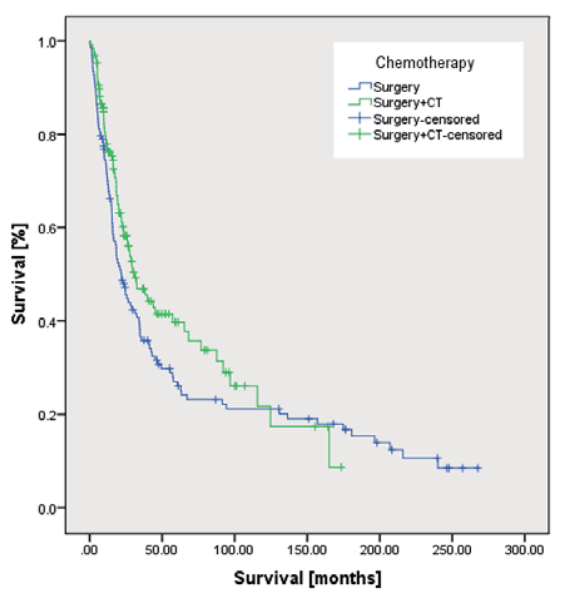

TABLE 2. Recorded reccurence patterns for T and N stage

\begin{tabular}{lccc}
\hline & $\begin{array}{c}\text { Peritoneal carcinomatosis } \\
\text { [n (\%)] }\end{array}$ & $\begin{array}{c}\text { Haemathogenous spread } \\
\text { [n (\%)] }\end{array}$ & $P$ \\
\hline T stage & & & \\
T1 & $0(0)$ & $0(0)$ & 0.019 \\
T2 & $1(8.3)$ & $4(36.4)$ & \\
T3 & $5(41.7)$ & $6(54.5)$ & \\
T4 & $6(50)$ & $1(9.1)$ & \\
N stage & & $1(9.1)$ & NS \\
N0 & $2(16.7)$ & $2(18.2)$ & $6(54.5)$ \\
N1 & $1(8.3)$ & $1(9.1)$ & $1(9.1)$ \\
N2 & $3(25)$ & $4(33.3)$ & \\
N3a & $2(16.7)$ & \\
N3b & &
\end{tabular}

NS = non-significant
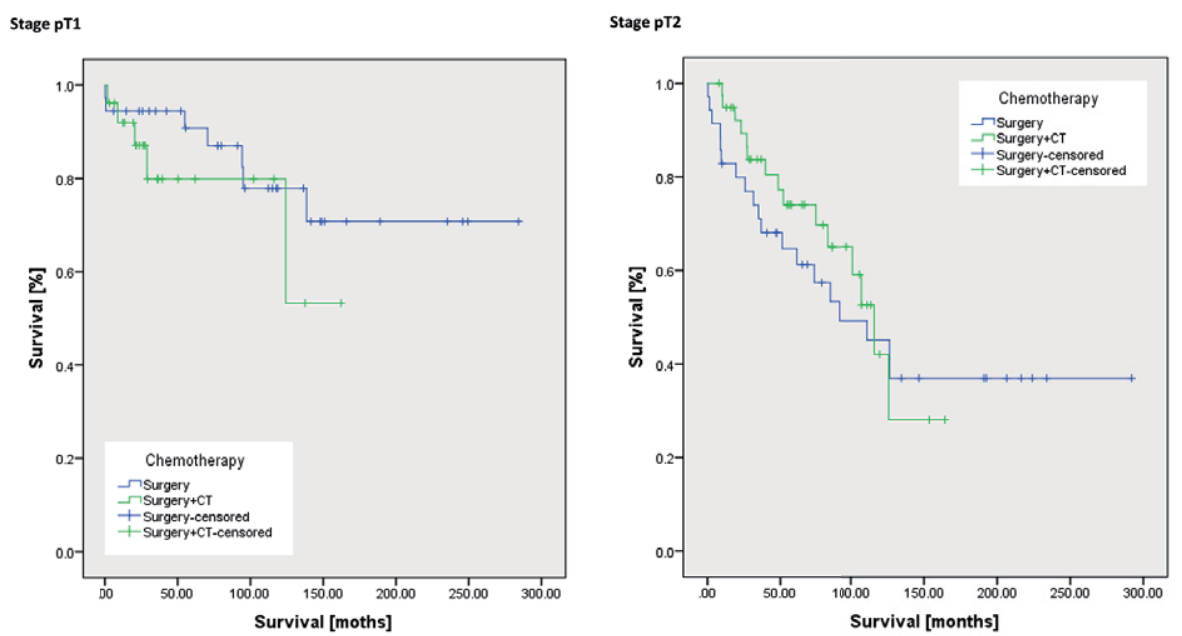

Stage PT4a

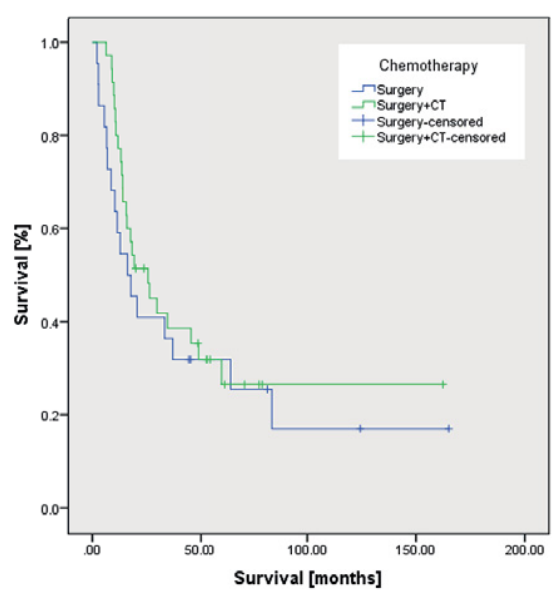

Stage pT4b

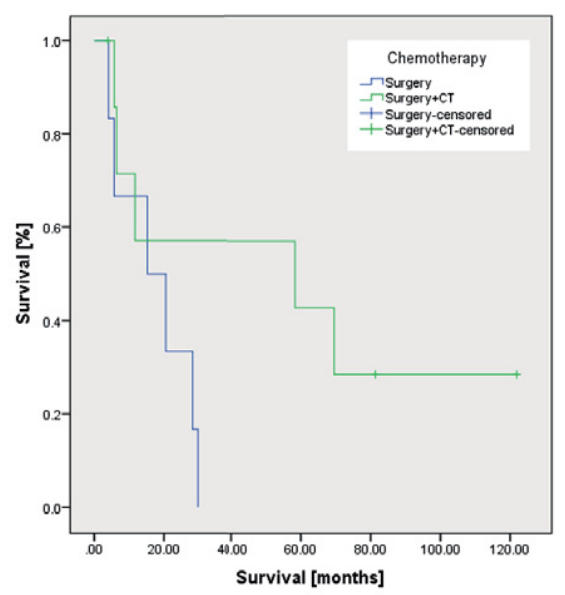

FIGURE 2. Overall survival of patients in Surgery compared to Surgery with chemotherapy group in different TNM T stages. 
TABLE 3. Predictors of survival

\begin{tabular}{lcccc} 
& \multirow{2}{*}{ Significance } & HR & \multicolumn{2}{c}{$95.0 \% \mathrm{Cl}$ for HR } \\
\cline { 4 - 5 } & & & Lower & Upper \\
\hline Age & 0.000 & 1.024 & 1.011 & 1.037 \\
CA 19-9 & 0.016 & 1.000 & 1.000 & 1.000 \\
UICC stage & 0.000 & 2.147 & 1.677 & 2.748 \\
Positive lymph nodes & 0.000 & 1.030 & 1.017 & 1.044 \\
Perioperative therapy & 0.032 & 0.741 & 0.563 & 0.975 \\
\hline
\end{tabular}

$\mathrm{HR}=$ hazard ratio revealed that only patients with the TNM stages T3 $(p=0.028)$ (Figure 2$), N 2(p=0.009)($ Figure 3$), N 3 b$ $(p=0.043)$ significantly benefit from perioperative and adjuvant treatment (Figure 3). For UICC stages survival analysis revealed significance for IIIa $(\mathrm{p}=$ $0.003)$ and IIIc $(p=0.03)$ but not for IIIb.

The multivariate analysis identified age, tumour marker Ca 19-9, UICC stage, number of positive lymph nodes and perioperative and adjuvant treatment as significant predictors (Table 3). Patients who received perioperative and adjuvant treatment had HR 0.741 (95\% CI: 0.563-0.975) compared to patients in the surgery only group.

The overall survival was significantly higher in the perioperative and adjuvant treatment group ( $\mathrm{p}$ $=0.038$ ) (Figure 1 ). The median survival in the surgery only group was 34.9 months compared to 51.7 months in the perioperative and adjuvant treatment group. The overall 5-year survival was $41.8 \%$ in the surgery only and $50.5 \%$ in the perioperative and adjuvant treatment group. The subgroup analysis

\section{Discussion}

The long-term results of the Dutch trial and more recently the results of the prospective randomised Taipei trial clearly confirmed the benefits of D2 lymphadenectomy in gastric cancer treatment. ${ }^{20,21}$
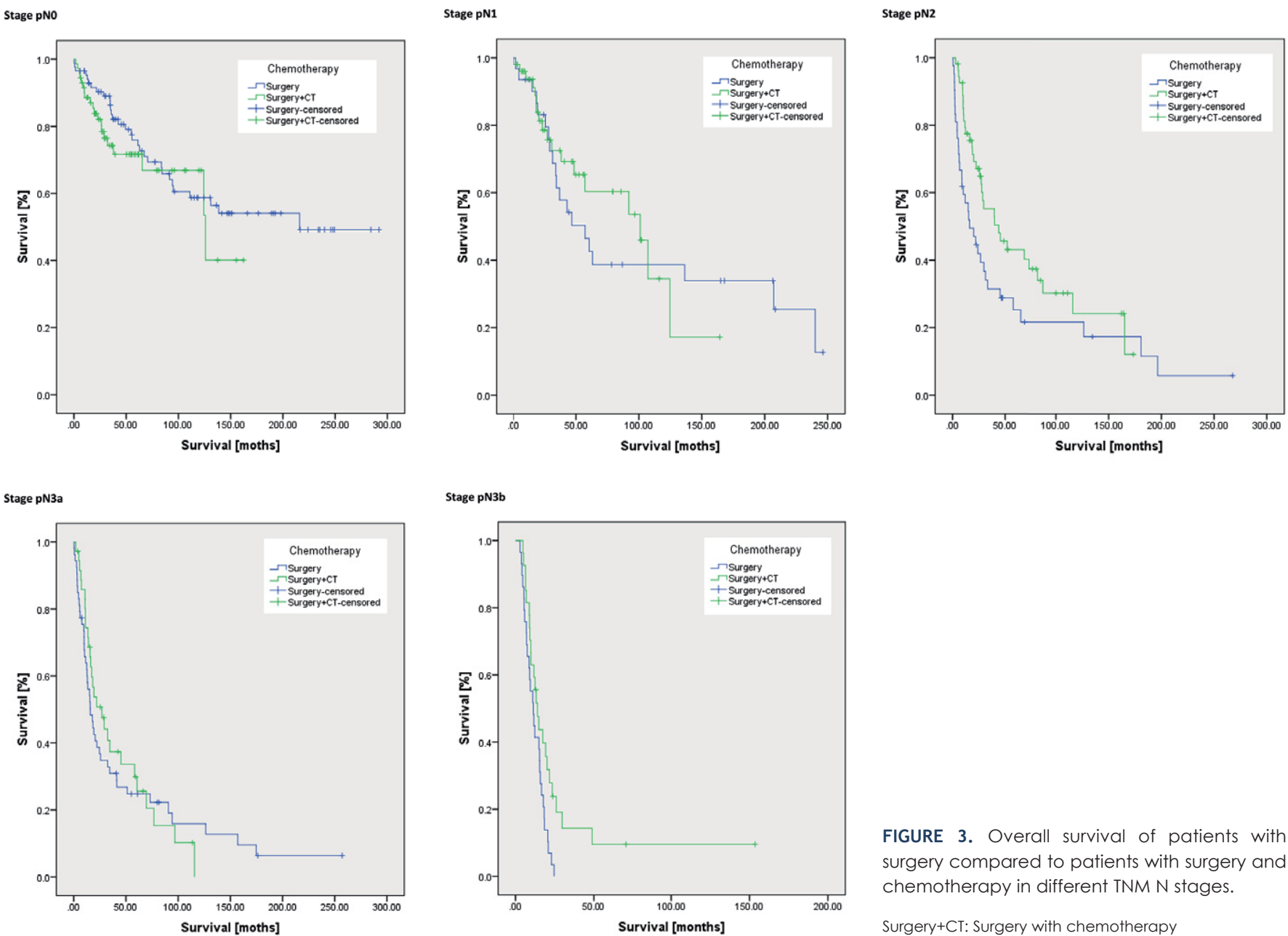

FIGURE 3. Overall survival of patients with surgery compared to patients with surgery and chemotherapy in different TNM N stages.

Surgery+CT: Surgery with chemotherapy 
In order to evaluate the effects of any adjuvant treatment in an intention to treat cohort, the results should only be evaluated in the light of a standardised extensive surgery. In the present study, we compared the results of radically resected gastric cancer patients with a D2 lymphadenectomy from single European centre to their propensity score matched counterparts treated with perioperative radio-chemotherapy or chemotherapy.

In our centre, D2 lymphadenectomy is considered standard treatment. To ensure a high level of surgical quality, gastric cancer patients are treated by a group of five specialised surgeons. The included patients in the present study had a D2 lymphadenectomy performed in $92.9 \%$, which is comparable to trials conducted in the East. The proportion of D2 lymphadenectomy resections in CLASSIC, ACTS-GS, ARTIST trials were $93 \%$ to $100 \%$. ${ }^{6,13}$ The number of D2 lymphadenectomy procedures in our study was superior to the majority of Western studies. The proportion of D2 resection was less than $10 \%$ in the MacDonald trial, while less than $40 \%$ of patients in the MAGIC and FNCLCC study had a D2 lymphadenectomy., 4,14 Even in a more recent European study ITACA-S, where the D2 lymph node dissection was the main goal of the study, a D2 lymphadenectomy was performed in $72 \%{ }^{12}$

The results in our analysis show the additional value of chemotherapy. The sufficient lymph node dissection allowed to evaluate the effect of multimodal treatment after D2 lymphadenectomy. Since 2003, when perioperative and adjuvant treatment was established for gastric cancer, many chemotherapy protocols were used; however, two types of perioperative and adjuvant treatment protocols dominated: perioperative radio-chemotherapy with capecitabine, and perioperative chemotherapy with epirubicin, oxalliplatin and capecitabine. While the latter protocols have recently been replaced by the FLOT (fluorouracil, leucovorin, oxaliplatin, docetaxel) protocol, the EOX and capecitabine protocols were applied in more than half of patients in the present study, with the remaining protocols being used in less than $10 \%$. Therefore, the results mainly apply to EOX and capecitabine treatment. We could show that the tolerance to these two treatments was very high. From the patients that received neoadjuvant treatment, $76.3 \%$ proceeded to surgery. From the patients who received adjuvant treatment, 93\% completed the therapy, while the remaining patients could not complete the treatment because of chemotherapy toxicity, poor general condition, tumour progres- sion and noncompliance. Therefore, a dropout of only $29 \%$ was recorded due to treatment toxicity. These results compare favourably to other trials. MacDonald et al. reported a 36\% rate of toxicity, while Sakuramoto et al. reported that only $65 \%$ of patients were able to finish the one-year adjuvant treatment with S1.6,14 Most of other studies also report a dropout of $30 \%$ to $40 \% .^{1-12}$ It seems that EOX and capecitabine stood out as excellently tolerated.

One of the rationales for perioperative treatment is the preoperative tumour downsizing and a higher $\mathrm{R} 0$ rate. In our study, a response was achieved in $12 \%$. Al-Bosse et al. reported response rates of $16 \%$ in the FLOT4 trial. ${ }^{10}$ Anderson et al. reported complete response in $6 \%$ of patients, with a downsizing noted in $17 \%$ in the MRC OE05 trial. ${ }^{11}$ In contrast to these studies where node negative patients were also counted as tumour downsizing, these patients were not included in our estimation of tumour downsizing. Hence, we believe that our estimation of tumour downsizing might be an underestimation. Nevertheless, these results are in concordance with other studies that used similar perioperative protocols to ours. These results confirmed that a downsizing of at least $12 \%$ can be expected with our perioperative treatment, further increasing the rates of resectability.

While the preoperative effects of radio-chemotherapy/chemotherapy might be undisputed, many opponents of preoperative treatment claim that preoperative treatment could increase perioperative morbidity and mortality due to chemo/ radio therapy damaged microcirculation. ${ }^{9}$ In our study, the comparison of perioperative morbidity did not confirm any significant correlation to perioperative treatment. Patients in the perioperative treatment group did not suffer from more surgical complications, nor did they experience more general complications as a result of general fatigue after perioperative treatment. The perioperative mortality in the perioperative group was at $0.4 \%$, even less than in surgery only group $(2.2 \%)$. These results indicate that the fears from perioperative treatment causing greater morbidity and mortality might be unsubstantiated.

The median survival in the perioperative and adjuvant treatment group was significantly longer than in surgery only group (51.7 months vs. 34.9 months; $\mathrm{p}=0.038$ ). The overall 5-year survival was $50.5 \%$ in the perioperative group and $41.8 \%$ in the surgery only group. Most of the studies evaluating perioperative treatment similarly support that perioperative and adjuvant treatment prolongs overall and disease survival. ${ }^{1-12}$ Our study addi- 
tionally confirms that perioperative and adjuvant treatment is beneficial after D2 lymphadenectomy. In the subgroup analysis, we detected a correlation between TNM stage and overall survival. However, the subgroup analysis only confirmed a survival benefit for perioperative and adjuvant treatment in stages T3, N2 and N3b. These results are not surprising, since they clearly point to the effects of D2 lymphadenectomy. Stages N0 to N1 can be completely cured with a radical lymph node dissection of the first and second tier; but once the lymph nodes have spread beyond the second tier, a systemic dissemination is highly likely. Therefore, patients with stages N2 and higher benefit from the addition of systemic treatment. Similar results were published by Zang et al. who showed that even the extension of lymphadenectomy beyond the second tier could not increase survival in patients with N3 disease. ${ }^{22}$ On the other hand, tumours that spread beyond the serosal layer (T4a and T4b) disseminate by direct shedding of tumour cells into peritoneal cavity. This is supported by the recurrence patterns in our autopsied patient cohort (Table 3). Patients with $\mathrm{T} 1$ to $\mathrm{T} 3$ tumours mainly recurred with haematogenous spread, while $\mathrm{T} 4 \mathrm{a}$ and $\mathrm{T} 4 \mathrm{~b}$ predominantly recurred in the peritoneal cavity. The effects of perioperative treatment were negated by the higher rate of intraperitoneal recurrence in stages T4a and T4b. It has been shown in the ACTS-GS trial that capecitabine or epirubicine, oxaliplatine and capecitabine cannot prevent the intraperitoneal recurrence. ${ }^{2}$ We have made the same observations that the regimens containing capecitabine or epirubicin, oxaliplatine and capecitabine are ineffective in patients with stages T4a and T4b where peritoneal recurrence is more prevalent.

The main limitation of our study is its retrospective nature. Although it was sufficiently powered and balanced with propensity score matching, we still must be cautious when interpreting the results. Although patients have been operated by the same group of experienced surgeons, and the surgical strategy and technique did not change since the beginning of the study period, comparison of patients from different time periods inevitably brings a certain bias to the analysis. Another drawback is the use of heterogenic perioperative regimens in our study. It is therefore difficult to determine which regimen has the best effect for D2 operated gastric cancer patients. Perhaps this question should be answered in a future study that will compare the effects of different subgroups of regimens to surgery only control group. Finally, the surgical procedures were performed in a highly specialized gastric cancer centre with five dedicated surgeons. Therefore, questions could be raised about generalisation of study findings with an extension of these results to other centres with lower caseloads.

In conclusion, our study results support the use of perioperative and adjuvant treatment in radically resected gastric cancer patients after D2 lymphadenectomy in stages IIIa and IIIc. The effects of perioperative and adjuvant treatment could be negated by the effects of the radical surgery in lower stages and by the biology of the disease in higher stages.

\section{References}

1. Cats A, Jansen EPM, Grieken NCT, Sikorska K, Lind P, Nordsmark M, et al. Chemotherapy versus chemoradiotherapy after surgery and preoperative chemotherapy for resectable gastric cancer (CRITICS): an international, open-label, randomised phase 3 trial. Lancet 2018; 19: 616-28. doi: 10.1016/S1470-2045(18)30132-3

2. Noh SH, Park SR, Yang HK, Chung HC, Chung IJ, Kim SW, et al. Adjuvant capecitabine plus oxaliplatine for gastric cancer after D2 gastrectomy (CLASSIC): 5-year follow-up of an open-lable, randomised phase 3 trial. Lancet 2014; 15: 1389-96. doi: 10.1016/S1470-2045(14)70473-5

3. Reece-Smith AM, Saunders JH, Soomro IN, Bowman CR, Duffy JP, Kaye PV, et al. Postoperative survival following perioperative MAGIC versus neoadjuvant OE02-type chemotherapy in oesophageal adenocarcinoma. Ann R Coll Surg Eng/ 2017; 99: 378-84. doi: 10.1308/rcsann

4. Cunningham D, Starling $N$, Rao $S$, Iveson $T$, Nicolson $M$, Coxon $F$, et al. Capecitabine and oxaliplatin for advanced esophagogastric cancer. $N$ Engl J Med 2008; 358: 36-46. doi: 10.1056/NEJMoa073149

5. Yehou M, Boige V, Pignon JP, Conroy $T$, Bouche $\mathrm{O}$, Lebreton $\mathrm{G}$, et al. Perioperative chemotherapy compared with surgery alone for resectable gastroesophageal adenocarcinoma: An FNCLCC and FFCD multicentre phase III trial. J Clin Oncol 2011; 29: 1715-21. doi: 10.1200/JCO.2010.33.0597

6. Sakuramoto S, Sasako M, Yamaguchi T, Kinoshita T, Fujii M, Nashimoto A, et al. Adjuvant chemotherapy for gastric cancer with S-1, an oral fluoropyrimidine. N Eng/ J Med 2007; 357: 1810-20. doi: 10.1056/NEJMoa0722

7. Nakajima T, Fujii M. What make differences in the outcome in the outcome of adjuvant treatments for resected gastric cancer? World J Gastroenterol 2014; 20: 11567-73. doi: 10.3748/wjg.v20.i33.11567

8. Toneto MG, Viola L. Current status of the multidisciplinary treatment of gastric adenocarcinoma. [English, Portuguese]. Arq Bras Cir Dig 2018; 31: 1-4. doi: 10.1590/0102-672020180001e1373

9. Chuang J, Gong J, Klempner SJ, Woo Y, Chao J. Refining the management of resectable esophagogastric cancer: FLOT4, CRITICS, OE05, MAGIC-B and the promise of molecular classification. J Gastrointest Oncol 2018; 9: 560-72. doi: 10.21037/jgo.2018.03.01

10. Bose K, Franck C, Müller MN, Canbay A, Link A, Venerito M. Perioperative therapy of oesophagogastric adenocarcinoma and future directions. Gastroenterol Res Pract 2017; 1-6. doi: 10.1155/2017/5651903

11. Alderson D, Cunningham D, Nankivell M, Blazeby JM, Griffin SM, Crellin A. Neoadjuvant cisplatin and fluorouracil versus epirubicin, cisplatin, and capecitabine followed by resection in patients with oesophageal adenocarcinoma (UK MRC OE05): an open-label, randomised phase 3 trial. Lancet 2017: 18: 1249-60 doi: 10.1016/S1470-2045(17)30447-3

12. Bajetta E, Floriani I, Di Bartolomeo M, Labianca R, Falcone A, DiCostanzo $\mathrm{F}$, et al. Randomized trial on adjuvant treatment with FOLFIRI followed by docetaxel and cisplatin versus 5-fluorouracil and folinic acid for radically resected gastric cancer. Ann Oncol 2014; 25: 1373-78. doi: 10.1093/annonc/ mdu146 
13. Park SH, Sohn TS, Lee J, Lim DH, Hong ME, Kim KM, et al. Phase III Trial to compare adjuvant chemotherapy with capecitabine and cisplatin versus concurrent chemoradiotherapy in gastric cancer: final report of the adjuvant chemoradiotherapy in stomach tumors trial, including survival and subset analyses. J Clin Oncol 2015; 33: 3130-3136. doi: 10.1200/JCO.2014.58.3930

14. Macdonald JS, Smalley SR, Benedetti J, HundahI SA, Estes NC, Stemmermann $\mathrm{GN}$, et al. Chemoradiotherapy after surgery compared with surgery alone for adenocarcinoma of the stomach or gastroesophageal junction. $N$ Engl J Med 2001; 345: 725-30. doi: 10.1056/NEJMoa010187

15. Japanese Gastric Cancer Association. Japanese gastric cancer treatment guidelines 2010 (ver. 3). Gastric Cancer 2011; 14: 113-23. doi: 10.1007/ s10120-011-0042-4

16. Oblak I, Skoblar Vidmar M, Anderluh F, Velenik V, Jeromen A, But Hadzic J. Capecitabine in adjuvant radiochemotherapy for gastric adenocarcinoma. Radiol Oncol 2014; 48: 189-96. doi: 10.2478/raon-2013-0065

17. Lee KY, Noh SH, Hyung WJ, Lee JH, Lah KH, Choi SH, et al. Impact of splenectomy for lymph node dissection on long-term outcome in gastric cancer. Ann Surg Oncol 2001; 8: 402-6. PMID: 11407513

18. Rosenbaum PR, Rubin DB. The central role of the propensity score in observational studies for causal effects. Biometrics 1983; 70: 41-55.

19. Li M. Using the propensity score method to estimate causal effects: a review and practical guide. Organ Res Methods 2012; 00: 1-39. doi: $10.1177 / 1094428112447816$

20. Hartgrink $\mathrm{HH}$, van de Velde $\mathrm{CJH}$, Putter $\mathrm{H}$, Bonekamp JJ, Kranenbarg $\mathrm{K}$ Songun I, et al. Extended lymph-node dissection for gastric cancer: who may benefit? Final results of the randomized Dutch Gastric Cancer Group trial. J Clin Oncol 2004; 22: 2069-77. doi: 10.1200/JCO.2004.08.026

21. Wu CW, Hsiung CA, Lo SS, Hsieh MC, Chen JH, Li AFY, et al. Nodal dissection for patients with gastric cancer: a randomized control trial. Lancet Oncol 2006; 7: 309-15. doi: 10.1016/S1470-2045(06)70623-4

22. Zhang $\mathrm{Y}$, Tian S. Does D2 plus para-aortic nodal dissection surgery offer a better survival outcome compared to D2 surgery only for gastric cancer consistently? A definite result based on a hospital population of nearly two decades. Scand J Surg 2013; 102: 251-7. doi: 10.1177/1457496913491343 